$\mathrm{Gl}$ ass Transi ti on and its Char act er i st i c Length for Thi $n$ Crossl inked Pol ystyrene Shel Is of Rodl i ke Capsul es

\begin{tabular}{|c|c|}
\hline 著者 & $\begin{array}{l}\text { SASAKI TAKASH, M SU MANABU, SHI NADA TAKAYUKN, } \\
\text { TERAMOTO M TSUHI RO }\end{array}$ \\
\hline $\begin{array}{l}\text { jour nal or } \\
\text { publ i cat i on titl e }\end{array}$ & $\begin{array}{l}\text { JOURNAL OF POLYMER SCI ENCE PART B POLYMER } \\
\text { PHYSI CS }\end{array}$ \\
\hline vol une & 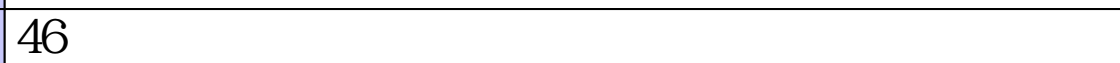 \\
\hline nunber & 19 \\
\hline page $r$ ange & $2116-2125$ \\
\hline year & $2008-10-01$ \\
\hline URL & ht t p: //hdl . handl e. net /10098/2042 \\
\hline
\end{tabular}




\title{
Glass Transition and its Characteristic Length for Thin Crosslinked Polystyrene Shells of Rodlike Capsules
}

\author{
TAKASHI SASAKI, MANABU MISU, TAKAYUKI SHIMADA, MITSUHIRO \\ TERAMOTO
}

Department of Materials Science \& Engineering, University of Fukui, Bunkyo, Fukui 910 8507, Japan

\begin{abstract}
Rodlike capsules consisting of a calcium carbonate core and a crosslinked polystyrene shell were synthesized, and the glass transition temperature $\left(T_{\mathrm{g}}\right)$ and characteristic length of the glass transition $\xi\left(T_{\mathrm{g}}\right)$ for the thin outer shells were investigated by temperature-modulated differential scanning calorimetry. The shell thickness ranged from 20 to $129 \mathrm{~nm}$. The ratio of the $T_{\mathrm{g}}$ for the outer shell to the bulk $T_{\mathrm{g}}$ increases with decreasing shell thickness $d$. The $d$-dependence of $T_{\mathrm{g}}$ is interpreted in terms of a simple two-layer model which assumes that an immobile layer exists near the core-shell interface. Shells of hollow capsules unexpectedly exhibit a similar $d$-dependence of $T_{\mathrm{g}}$ to that for the filled capsules. This is characteristic of the crosslinked polymeric shells, and is attributed to certain spatial heterogeneity of crosslink distribution, and/or to the unstable configuration in the ultrathin shell that does not undergo relaxation due to the crosslink. The latter idea is based on the assumption that unstable configurational state is responsible for the $T_{\mathrm{g}}$ shift from the bulk value observed for nanosized polymeric materials. The ratio of the characteristic length for the shell of the filled capsule to that of the bulk $\xi_{\mathrm{f}}\left(T_{\mathrm{g}}\right) / \xi_{\mathrm{b}}\left(T_{\mathrm{g}}\right)$ decreases with decreasing $d$. The results are interpreted in terms of the configurational entropy, and it is also suggested that the configurational state of network polymer chains in the shell affects the characteristic length.
\end{abstract}

Keywords: core/shell and hollow capsules; polystyrene; glass transition; characteristic length; TMDSC

Correspondence to: T. Sasaki (E-mail: sasaki@matse.u-fukui.ac.jp)

\section{INTRODUCTION}

Fabrication of nanosized polymeric capsules has an intense interest because they can be used as suitable materials for such as drug delivery systems and nano-reactors. Capsules possessing polymeric ultrathin outer shells have been synthesized by various methods, ${ }^{1-5}$ and especially shell crosslinked capsules are important for practical 
applications because of their stability. ${ }^{1,2,4,6}$ However, the thermal and mechanical properties of nanocapsules have not been thoroughly investigated so far. In a recent decade, on the other hand, anomalous dynamics of nanosized polymers which is very different from that in a bulk state has been attracted considerable attention, especially for ultrathin polymer films. ${ }^{7,8}$ It is reasonable to expect that ultrathin shells in polymeric capsules also exhibit different segmental dynamics from that in the bulk state.

A crucial problem concerning the anomalous dynamics in nanosized polymeric materials lies in the lack of uniformity in the glass transition behaviors depending strongly on the systems: for some polymers, the glass transition temperature $\left(T_{\mathrm{g}}\right)$ of supported films thinner than ca. $60 \mathrm{~nm}$ may increase or decrease with decreasing thickness depending on the substrate, while for free-standing polystyrene films $T_{\mathrm{g}}$ decreases with decreasing thickness. ${ }^{7,9-15}$ Additionally, glass formers confined in nanosized pores exhibit different $T_{\mathrm{g}} \mathrm{S}$ (or dynamics) from the bulk, ${ }^{16,17}$ while polystyrene nanospheres exhibit no $T_{\mathrm{g}}$ shift from the bulk value. ${ }^{18,19}$ To explain the thickness dependence of $T_{\mathrm{g}}$ for ultrathin films, various models have been proposed, but it seems to be rather difficult to find a universal explanation for the above assorted results. One possible explanation is based on the interface effects, ${ }^{20-24}$ assuming that a layer with different mobility from the bulk is supposed to exist near the interface, which can explain the thickness dependence of $T_{\mathrm{g}}$ for supported films. On the other hand, the effects of different configurations from the bulk (or confinement effects) should also be considered. $^{25,26}$ As another approach, the dynamic percolation model, which is an extension of the free-volume model, has been proposed. ${ }^{27}$

In a previous paper, we have investigated the glass transition temperature of thin outer shells of rodlike capsules, which are composed of a crosslinked polystyrene shell and a calcium carbonate core. ${ }^{6}$ The shell thickness ranged from 26 to $81 \mathrm{~nm}$. The observed $T_{\mathrm{g}}$ of the shell has been revealed to be higher than the bulk value. The results strongly suggest the interface effect on $T_{\mathrm{g}}$ : it is likely that polymer segments are immobilized at the core/shell interface due to an interaction between the polymer and the core material. However, the results also suggest that the $T_{\mathrm{g}}$ deviation reflects contribution from the configuration effect as well as the heterogeneous nature characteristic of the crosslinked polystyrene. Indeed, non-crosslinked polystyrene shells exhibit no $T_{\mathrm{g}}$ deviation from the bulk value. To understand the above behaviors of thin crosslinked shells, we should go further into the nature of glass transition phenomenon.

As for glass-forming liquids, dynamical heterogeneity induced by such as density 
fluctuations has been demonstrated, ${ }^{28-30}$ and the characteristic length $\xi$ of this heterogeneity at $T_{\mathrm{g}}$ is an important parameter to characterize the glass transition behavior. The values of $\xi\left(T_{\mathrm{g}}\right)$ can be evaluated by temperature-modulated differential scanning calorimetry (TMDSC). ${ }^{31,32}$ According to the Adam-Gibbs theory, ${ }^{33}$ segmental dynamics is characterized by cooperatively rearranging region (CRR) of which the size has strong temperature dependence. It is reasonably assumed that the length scale of CRR at $T_{\mathrm{g}}$ corresponds to $\xi\left(T_{\mathrm{g}}\right)$.

In this work, we further investigate the glass transition behaviors of crosslinked polystyrene ultrathin shells deposited on the surface of calcium carbonate whisker. We prepared core/shell and hollow capsules with various shell thicknesses and degrees of crosslink, and evaluate thickness dependence of both $T_{\mathrm{g}}$ and $\xi\left(T_{\mathrm{g}}\right)$ for the outer shells by step-scan heating measurements of DSC and TMDSC cooling scans, respectively. The results reveal unambiguous thickness dependence of the parameters, which are interpreted in terms of interface effects and of the unstable configuration effects for crosslinked polymers.

\section{EXPERIMENTAL}

\section{Preparation of capsules}

Rodlike capsules consisting of a calcium carbonate core (Maruo Calcium Co. BS-P) and a crosslinked polystyrene shell were prepared according to the method described in the previous papers. ${ }^{6,34}$ Radical copolymerization of styrene and divinylbenzene (DVB) in the presence of suspended BS-P with laurylbenzenesulfonic acid was performed. We also added a small amount of coupling reagent, methacrylic acid 3-(tri-methyoxysilyl)propyl ester (MAMSP) in the reaction mixture. Styrene and DVB was distilled under reduced pressure before use. The distilled DVB still contained impurities such as ethyl vinylbenzene, ${ }^{35}$ but was used without further purification. The purity of DVB was determined by gas chromatography. Taking into account the obtained purity of DVB, we evaluated the degree of crosslink $X_{\mathrm{c}}$, which is defined by

$$
X_{\mathrm{C}}=m_{\mathrm{DVB}} /\left(m_{\mathrm{S}}+m_{\mathrm{DVB}}\right)
$$

where $m_{\mathrm{S}}$ and $m_{\mathrm{DVB}}$ are the net masses of styrene and DVB fed for the polymerization reaction. By varying the amount of monomers with respect to BS-P, we could obtain capsules with different shell thicknesses as we have reported previously. ${ }^{6}$ We prepared core/shell capsules with various $X_{\mathrm{c}} \mathrm{s}$ and shell thicknesses as listed in Table 1. Hollow capsules were also prepared from the obtained core/shell capsules by dissolving the core in hydrochloric acid. ${ }^{34}$ Removal of the core was confirmed by FT-IR spectroscopy and wide angle X-ray diffraction (absence of diffraction signals due to $\mathrm{CaCO}_{3}$ ). The 
morphology of the obtained capsules was investigated by transmission electron microscopy (TEM) using a Jeol JEM-2000FXII operated with an acceleration voltage of $200 \mathrm{kV}$. Polymer content in the synthesized core/shell capsules was evaluated by volumetric analysis: a finite mass of core/shell sample was immersed in hydrochloric acid $(\mathrm{pH}=1)$ and the mixture was stirred for at least $12 \mathrm{~h}$ to dissolve the core completely. After removing water and hydrochloric acid by evaporation, residue was again dissolved in distilled water, and calcium ion content in this aqueous solution was determined by chelatometric titration. Reference bulk samples were prepared from the hollow capsules by annealing them at $140^{\circ} \mathrm{C}$ for $4 \mathrm{~h}$ under high vacuum. We made sure by TEM that the hollow capsules were well melted after the above annealing, and can be used as bulk samples.

\section{Calorimetry}

DSC measurements were executed by using a Perkin Elmer Pyris Diamond DSC calorimeter. An ice-water bath was used as a cooling system. Sample temperature and heat flow were calibrated by using an indium standard, and observed heat capacity was calibrated by using a sapphire standard. Glass transition temperature was evaluated from the reversing heat capacity trace, which was obtained by step-scan mode of DSC. ${ }^{6}$ Each step of the step-scan was consisted of a heating stage of $2 \mathrm{~K}$ at $5 \mathrm{~K}$ $\min ^{-1}$ and a temperature holding stage which ended when 10 consecutive data points fell within $\pm 0.01 \mathrm{~mW}$. Heat capacity of the calcium carbonate whisker (BS-P) was also measured by the above step-scan method.

To evaluate the characteristic length $\xi\left(T_{\mathrm{g}}\right)$, we performed cooling scans of TMDSC. ${ }^{31,32,36}$ The sample was first heated at $140^{\circ} \mathrm{C}$ for $2 \mathrm{~min}$, and a temperature-modulated cooling scan was executed: a saw-tooth modulation with an amplitude of $0.5 \mathrm{~K}$ and a period of $60 \mathrm{~s}$ was applied to an underlying cooling scan at $-0.2 \mathrm{~K} \mathrm{~min}^{-1}$. We made sure from the Lissajous plots that nonlinear effect of thermal response is negligible for the present small amplitude of temperature modulation. ${ }^{37}$ For the hollow capsules, the above TMDSC measurements could not provide reliable data because the capsules were found to be collapsed after the TMDSC scan, in which the sample had been heated for more than $100 \mathrm{~min}$ above $T_{\mathrm{g}}$. Thus, we could not evaluate $\xi\left(T_{\mathrm{g}}\right)$ for the hollow shells. On the other hand, the core/shell capsules did not undergo any morphological change even after the present TMDSC scan. We confirmed for the core/shell samples that two sets of data obtained by repeating the TMDSC scan for the same specimen coincide with each other within the experimental error, indicating that the calorimetric nature of the core/shell capsule does not change at 
least after one TMDSC scan.

The complex heat capacity $C^{*}(\omega)$ was evaluated via Fourier transformation of the observed heat flow data from TMDSC, where $\omega$ is the modulation frequency. ${ }^{31}$ The storage and loss heat capacities $C^{\prime}(\omega)$ and $C^{\prime \prime}(\omega)$ are given by

$$
\begin{aligned}
& C^{\prime}(\omega)=\left|C^{*}(\omega)\right| \cos \phi \\
& C^{\prime \prime}(\omega)=\left|C^{*}(\omega)\right| \sin \phi
\end{aligned}
$$

where $\phi$ is the phase shift between the heat flow and temperature. We extracted the first harmonic of $C^{\prime}(\omega)$ and $C^{\prime \prime}(\omega)$ for each modulation cycle. Typical result is presented in Figure 1, where $C^{\prime}$ and $C^{\prime \prime}$ are plotted as a function of temperature (average temperature for each modulation cycle).

\section{Evaluation of the characteristic length}

The characteristic length of the glass transition $\xi\left(T_{\mathrm{g}}\right)$ was evaluated according to the fluctuation dissipation theory. ${ }^{32}$ The characteristic length is approximately given by

$$
\xi=\left[\frac{k T^{2} \Delta\left(1 / C_{V}\right)}{\rho(\delta T)^{2}}\right]^{1 / 3}
$$

with

$$
\Delta\left(1 / C_{V}\right) \approx 1 / C_{p}(\mathrm{~g})-1 / C_{p}(\mathrm{l})
$$

where $\rho$ is the mass density, $C_{p}(\mathrm{~g})$ and $C_{p}(\mathrm{l})$ are the molar heat capacities at constant pressure in the glassy and liquid states, respectively, and $\delta T$ is the temperature fluctuation parameter (root mean-square temperature fluctuation per average CRR). $\Delta\left(1 / C_{p}\right)$ was evaluated from the reversing heat capacity data obtained by the step-scan heating measurements. As for the core/shell capsules, the heat capacity data were corrected by using the polymer content in the capsule (from the volumetric analysis) as well as by using the heat capacity data for calcium carbonate whisker (from the step-scan DSC). $\quad \delta T$ was evaluated from the observed profile of $C^{\prime \prime}(T)$ by fitting with a Gaussian distribution function as

$$
C^{\prime \prime}(T)=A \exp \left\{-\frac{\left[\left(T-T_{\mathrm{g}}(\omega)\right]^{2}\right.}{2(\delta T)^{2}}\right\}
$$

where $T_{\mathrm{g}}(\omega)$ is the temperature at which $C^{\prime \prime}(T)$ exhibits the maximum. The temperature $T_{\mathrm{g}}(\omega)$ was found to be a few degrees higher than the $T_{\mathrm{g}}$ obtained from the step-scan DSC. This indicates that the time scale of the current TMDSC $(\omega=0.105$ rad s${ }^{-1}$ ) is shorter than that of the step-scan DSC. 


\section{RESULTS AND DISCUSSION Morphology}

Table 1 shows the thickness of the shell for the samples used in this study. Hollow particles were successfully obtained. As we have demonstrated in the previous paper, the core removal process occurs via transportation of $\mathrm{Ca}^{2+}$ ion through the polystyrene shell, which can take place even if there is no defect in the shell. ${ }^{6}$ Table 1 shows that the thickness of the hollow shell $d_{\mathrm{h}}$ is greater than that of the shell of the filled core/shell capsule (referred to as filled shell henceforth) $d_{\mathrm{f}}$. We found that such thickening is associated with shrinkage of the capsule as evidenced by the reduction in the diameter. As we have discussed previously, the above thickening is due to structural relaxation which occurs upon removal of the core, and this suggests that the configuration in the thin shell in the presence of the core is far from an equilibrium state. ${ }^{34}$ Figure 2 shows the ratio $d_{\mathrm{h}} / d_{\mathrm{f}}$ plotted against $d_{\mathrm{f}}$, indicating that the shell thickening becomes prominent as the original thickness decreases. This suggests that the thinner the shell, the less stable the as-prepared configurational state. On the other hand, the ratio $d_{\mathrm{h}} / d_{\mathrm{f}}$ exhibits no clear dependence on $X_{\mathrm{c}}$, suggesting that crosslink does not impede significantly the thickening. However, the unstable configuration seems to be retained to some extent in the hollow shell due to crosslink as will be discussed later.

Figure 3 shows typical transmission electron micrographs for the core/shell and hollow capsules. The outer shells are successfully formed, but the surface of the shell is rather rough. Such morphology may be related to the polymerization mechanism. Two possible mechanisms are assumed: (1) in the presence of surfactant, thin monomer layer is formed on the surface of suspended whisker particles, and then, radical polymerization proceeds in the layer. (2) Polymerization occurs in microemulsion micell independent of the suspended whisker particles, yielding microspheres. Then, the microspheres are deposited onto the whisker surface to form a shell with ragged surface. It is likely that both the two mechanisms occur for the present styrene-DVB system, but detailed mechanism is now under investigation.

We also found that the yielded shell thickness is approximately proportional to the relative amount of monomers fed in the reaction with respect to BS-P. Comparing the samples with different $X_{\mathrm{c}}$ for the same relative amount of fed monomers (samples A, C, $\mathrm{D}$, and $\mathrm{E}$ ), we see that the shell thickness tends to decrease with increasing $X_{\mathrm{c}}$. This might be partially due to reduction in the interfacial free energy between the monomer phase and calcium carbonate due to DVB. 


\section{Glass transition temperature}

Figure 4 shows the glass transition temperature for the reference bulk samples $T_{\mathrm{g}}{ }^{\mathrm{b}}$ obtained from the step-scan measurements. As expected, $T_{\mathrm{g}}{ }^{\mathrm{b}}$ increases with increasing

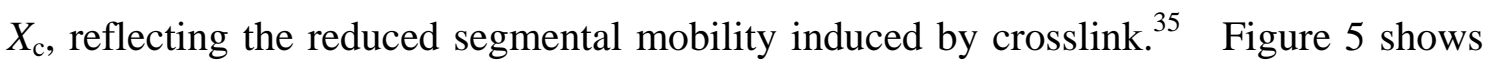
the ratio of the glass transition temperature of the filled shell to that of the corresponding bulk, $T_{\mathrm{g}}^{\mathrm{f}} / T_{\mathrm{g}}{ }^{\mathrm{b}}$ plotted against the shell thickness. Dividing $T_{\mathrm{g}}{ }_{\mathrm{f}}$ by $T_{\mathrm{g}}{ }^{\mathrm{b}}$ cancels contribution from the effects of varying $X_{\mathrm{c}}$. We see that the increasing feature of $T_{\mathrm{g}}$ from the bulk is enhanced as the thickness decreases, thus the size effect on $T_{\mathrm{g}}$ is demonstrated. As has been discussed for supported thin films, strong interaction at the polymer-substrate interface may be a cause for reduction in segmental mobility near the interface, ${ }^{11,20-24,38}$ though contradicting results have also been reported. ${ }^{39}$ One might suppose that the present increase in $T_{\mathrm{g}}$ is due to strong adsorption (anchoring) of polystyrene chains at the surface of BS-P. As we have reported previously, shells without a coupling reagent, MAMSP give smaller $T_{\mathrm{g}}$ shift from the bulk value, ${ }^{6}$ and thus, a core-shell interaction due to the presence of the coupling reagent might be expected. However, we infer that such core-shell interaction at the interface is not the main cause for the $T_{\mathrm{g}}$ behavior with respect to $d$ as we discuss later.

Based on the assumption of the immobile interfacial layer, we here employ a simple two-layer model consisting of an immobile layer near the interface and an upper layer with a normal mobility (in a bulk state) to analyze the profile in Figure 5. Assuming that the $T_{\mathrm{g}}$ of the whole shell is a simple arithmetic average of the $T_{\mathrm{g}}$ of each layer, ${ }^{12}$ the ratio $T_{\mathrm{g}}{ }^{\mathrm{f}} / T_{\mathrm{g}}{ }^{\mathrm{b}}$ for the two-layer model is given by

$$
T_{\mathrm{g}}^{\mathrm{f}} / T_{\mathrm{g}}^{\mathrm{b}}=1+\frac{A}{d}
$$

with

$$
A=\lambda\left(T_{\mathrm{g}}^{\mathrm{i}} / T_{\mathrm{g}}^{\mathrm{b}}-1\right)
$$

where $\lambda$ and $T_{\mathrm{g}}{ }^{\mathrm{i}}$ are the thickness and glass transition temperature of the immobile interface layer, respectively. Assuming that $A$ is independent of the shell thickness, least-squares fitting analysis gave $A=0.457 \mathrm{~nm}$ as the best-fit parameter. The dotted curve in Figure 5 indicates the fitting result.

Besides the immobile interface layer, we should also consider the contribution from the free-surface (polymer-air interface) where higher segmental mobility than the bulk is expected. Considerable research has revealed the existence of such mobile surface layer for various polymer systems. ${ }^{12,19,21,40-42}$ Considering a three-layer model consisting of a free-surface layer, a bulk-like interior part, and an interface layer, ${ }^{20}$ the parameter $A$ in eq 7 is to be interpreted as 


$$
A=\lambda\left(\frac{T_{\mathrm{g}}^{\mathrm{s}}+T_{\mathrm{g}}^{\mathrm{i}}}{T_{\mathrm{g}}^{\mathrm{b}}}-2\right)
$$

where $T_{\mathrm{g}}^{\mathrm{s}}$ is the glass transition temperature of the free-surface layer. Unfortunately, we could not evaluate $T_{\mathrm{g}}^{\mathrm{s}} / T_{\mathrm{g}}^{\mathrm{b}}$ and $T_{\mathrm{g}}^{\mathrm{i}} / T_{\mathrm{g}}^{\mathrm{b}}$ separately from the currently obtained results. Anyhow, the obtained parameter $A$ is considered to include the contribution from the mobile free-surface layer as well as the immobile interface layer.

As for the hollow capsules, the ratio $T_{\mathrm{g}}^{\mathrm{h}} / T_{\mathrm{g}}^{\mathrm{b}}$ exhibits a similar tendency to that of $T_{\mathrm{g}}^{\mathrm{f}}$ $/ T_{\mathrm{g}}^{\mathrm{b}}$ as shown in Figure 5 (filled squares). This implies that the glass transition temperature of the hollow shell is still higher than that of the corresponding bulk. This result is surprising because hollow shell is a counterpart of free-standing thin film having free surfaces on both sides, and is expected to exhibit higher mobility and lower $T_{\mathrm{g}}$. This inconsistency with the results of thin films may be related to the nature of crosslinked polymer shell, and we here propose two possible assumptions: (1) crosslink segments (DVB units) tend to localize at the core/shell interfacial region for some reasons such as the different reactivity of DVB from that of styrene, so that the interfacial region is highly crosslinked and remains immobile even without the core material (heterogeneous crosslink). It is likely that this effect overcomes the interfacial interaction effect (anchoring of the polystyrene chains at the interface), suggesting that the adsorption of the polystyrene chains at the interface is not strong, because the hollow shells exhibit a similar profile to that of the filled shells (Figure 5). (2) The confinement effect on $T_{\mathrm{g}}$ (effect of unstable configuration) is important, and the $T_{\mathrm{g}}$ is essentially governed by the shell thickness that embodies the confinement effect rather than by the status of the interface. As we mentioned in the foregoing section, the shell thickness increases after the removal of the core due to structural relaxation. However, crosslink may hinder the relaxation of altering the configuration to some extent, and therefore, the hollow shells may be essentially in a similar configurational state to that of the filled shells. Indeed, it is not reasonable to consider that two types of shell with different configurations obey the same $d$-dependence as shown in Figure 5.

Furthermore, we should note here that non-crosslinked polystyrene shells exhibit no $T_{\mathrm{g}}$ shift from the bulk value as we have shown in the previous papers. ${ }^{6,34}$ This may be explained based on the above assumption (2) as that crosslinked shells can retain unstable configurations that have been formed during the polymerization, due to the restriction imposed by the crosslink, while for non-crosslinked shells, relaxation occurs yielding more bulk-like configurations. In general, such structural relaxation may play an important role in the $T_{\mathrm{g}}$ shift for nanosized polymeric materials. The apparently 
non-uniform glass transition behaviors of nanosized polymeric materials reported so far might be explained in terms of the degree of configurational relaxation. For example, little $d$-dependence of $T_{\mathrm{g}}$ has been reported for crosslinked poly(vinyl chloride) thin films. ${ }^{43}$ However, in this case, crosslink has been introduced after the formation of the thin films, when the unstable configurational state may have already been released. On the other hand, it has been reported that thinly sliced epoxy resin ( $d=40-80 \mathrm{~nm})$ shows $T_{\mathrm{g}}$ depression from the bulk value that is associated with a broadening of $T_{\mathrm{g}}{ }^{44}$ In the case of highly crosslinked epoxy resin, the spatially heterogeneous crosslink effect may not be significant, and the $T_{\mathrm{g}}$ shift should be attributed to unstable configuration in a confined state, though the direction of the $T_{\mathrm{g}}$ shift is opposite to that of the present system.

The above assumption (2) may conflict with the assumption of the immobile interface layer. However, we should note that the latter assumption is supported by experimental results for various systems, ${ }^{20-22,24}$ and also for the current capsules by the effect of the coupling reagent mentioned above. Thus, we infer that the immobile interface layer probably exists for the present shells, but significance of this effect on $T_{\mathrm{g}}$ is still unknown.

In comparing the current results of the capsules with those of ultrathin polymer films, we should further consider the effect of geometry (curved and flat thin layers) on $T_{\mathrm{g}}$. Long and Lequeux have proposed a dynamic percolation model, which is based on the heterogeneous nature of dynamics that has been observed for glass-formers, and it considers critical percolation concentration of slow domains that are induced by density fluctuations. $^{27}$ The percolation threshold for a curved layer is considered to be lower than that of a flat layer, therefore, the size effect on $T_{\mathrm{g}}$ is supposed to be weaker for the current curved shells than for flat films, and the onset thickness below which the $T_{\mathrm{g}}$ practically deviates from the bulk value is supposed to be lower for the former.

\section{Characteristic length}

The characteristic length of the glass transition $\xi\left(T_{\mathrm{g}}\right)$ for the reference bulk sample is shown in Figure 4 (open squares). We see that $\xi\left(T_{\mathrm{g}}\right)$ decreases with increasing $X_{\mathrm{c}}$, which is consistent with our previous result. ${ }^{45}$ The values are lower than those obtained from the empirical method of conventional DSC using the rule of thumb. ${ }^{32,45}$ This tendency has been reported for various glass forming liquids, ${ }^{32}$ and might be due to uncertainty of the numerical factor used in the rule of thumb.

Table 2 shows values of $\xi\left(T_{\mathrm{g}}\right)$ for the filled shells. We evaluated glass transition temperature of the immobile interfacial region $T_{\mathrm{g}}{ }^{\mathrm{i}}$ according to the two-layer model 
using $A=0.457 \mathrm{~nm}$, assuming that the thickness of the interface layer $\lambda$ in eq 7 is equal to $\xi\left(T_{\mathrm{g}}\right)$. The results are shown in Table 2, indicating that $T_{\mathrm{g}}{ }^{\mathrm{i}}$ is almost linearly proportional to $X_{\mathrm{c}}$. We should note here a tricky problem that $A$ was estimated by assuming that it is independent of the shell thickness, while $T_{\mathrm{g}}{ }^{\mathrm{i}}$ in Table 2 was obtained from $A$ that depends on the shell thickness through the $d$-dependence of $\xi\left(T_{\mathrm{g}}\right)$. Anyhow, the $T_{\mathrm{g}}^{\mathrm{i}}$ values in Table 2 are to be regarded as rough estimations. Moreover, the ratio $T_{\mathrm{g}}^{\mathrm{i}} / T_{\mathrm{g}}{ }^{\mathrm{f}}$ increases with increasing $X_{\mathrm{c}}$ as listed in Table 2 . This means that the immobilization in the interfacial region is enhanced as the crosslink density increases. Based on the assumption (1) in the foregoing section (localization of the crosslink units near the interface), the result may be attributed to the enhancement of heterogeneity of crosslink for higher $X_{\mathrm{c}}$, which is consistent with the result of $\xi\left(T_{\mathrm{g}}\right)$ (Table 2).

Figure 6 shows the ratio of the characteristic length of the filled shells to that of the corresponding reference bulk $\xi_{\mathrm{f}}\left(T_{\mathrm{g}}\right) / \xi_{\mathrm{b}}\left(T_{\mathrm{g}}\right)$ plotted against $d_{\mathrm{f}}$. The ratio $\xi_{\mathrm{f}}\left(T_{\mathrm{g}}\right) / \xi_{\mathrm{b}}\left(T_{\mathrm{g}}\right)$ decreases from unity with decreasing $d_{\mathrm{f}}$, showing that the reduction of characteristic length from the bulk value becomes prominent for thinner shells. This indicates that the characteristic length scale of the glass transition for the filled shell is smaller than for the bulk, and that the $T_{\mathrm{g}}$ signal becomes broader for thinner shells.

For the decreasing feature of $\xi\left(T_{\mathrm{g}}\right)$ with increasing $X_{\mathrm{c}}$, we have proposed two possible origins, ${ }^{45}$ i.e., decrease in the configurational entropy $s_{\mathrm{c}}{ }^{*}$ of the smallest CRR at $T_{\mathrm{g}}$ with increasing $X_{\mathrm{c}}$, and the heterogeneous nature of crosslink. ${ }^{46-48}$ The latter includes contribution from the distribution of tie chain length between the crosslink units, and that from the spatial distribution of crosslink segment. However, the heterogeneity of crosslink for the filled shells may not be significantly different from the present bulk samples: the heterogeneity in the tie chain length does not change by converting the capsules to the bulk, and also no significant change in the spatial distribution of crosslink unit may be expected because of topological limitation of network structure. Therefore, it is likely that the observed $d$-dependence of the ratio $\xi_{\mathrm{f}}\left(T_{\mathrm{g}}\right) / \xi_{\mathrm{b}}\left(T_{\mathrm{g}}\right)$ is mainly governed by the effect of configurational state, which concerns the essential nature of network polymer systems.

Interpreting the characteristic length $\xi$ in terms of the CRR model of the Adam-Gibbs theory gives a relation ${ }^{45}$

$$
\xi^{3} \propto S_{\mathrm{C}}^{*} / S_{\mathrm{C}}
$$

where $S_{\mathrm{c}}$ is the configurational entropy of the macroscopic material. It is reasonable to assume that $S_{\mathrm{c}}$ decreases with decreasing $d_{\mathrm{f}}$ because of spatial limitation. Hence, the decrease in $\xi_{\mathrm{f}}\left(T_{\mathrm{g}}\right)$ with decreasing $d_{\mathrm{f}}$ means that $s_{\mathrm{c}}{ }^{*}$ decreases with decreasing $d_{\mathrm{f}}$, of 
which the tendency is stronger than that for $S_{\mathrm{c}}$. However, it may not be easy to understand intuitively the physical meaning of such strong thickness dependence of $s_{\mathrm{c}}{ }^{*}$.

In the context of the foregoing section, it would be worth trying to interpret the observed thickness dependence of $\xi_{\mathrm{f}}\left(T_{\mathrm{g}}\right)$ in terms of the two-layer model, assuming that different segmental dynamics near the interface from that of the bulk gives different characteristic length. However, we found that a simple two-layer model gives meaningless value of the fitting parameter; this is probably due to the inappropriate assumption for the nature of characteristic length that there is no coupling between the two layers. It is still important to construct a proper model that can predict the $d$-dependence of the characteristic length; this would also provide reasonable interpretation for the behavior of $s_{\mathrm{C}}{ }^{*}$ discussed above.

We found that the characteristic length decreases with increasing $X_{\mathrm{c}}$ both for the filled shells and the bulk samples. Thus, one might anticipate that the characteristic length has a direct connection with average distance between crosslink points. We estimated the average distance between the nearest neighboring crosslink units $L$ from the net content of DVB fed in the polymerization reaction as ${ }^{45}$

$$
L=2^{1 / 6} N_{\mathrm{c}}^{-1 / 3}
$$

where $N_{\mathrm{c}}$ is the number of crosslink units per unit volume. Figure 7 shows characteristic length plotted against $L$, indicating that characteristic length is lower than $L$, and a weak $L$-dependence of $\xi\left(T_{\mathrm{g}}\right)$ is observed both for the filled shells and the bulk samples. $^{49}$ This suggests that the distance $L$ does not directly determine the characteristic length as we have already demonstrated in the previous work for the bulk systems. $^{45}$ This confirms that the characteristic length scale in crosslinked polymers is determined rather by the essential nature of segmental dynamics in the network architecture, which has a close relation with the configurational state as well as with the heterogeneous nature of crosslink density.

\section{CONCLUSIONS}

In this paper, we have demonstrated that the $T_{\mathrm{g}}$ increases with decreasing $d$ for crosslinked polystyrene shells of rodlike capsules, and this can be interpreted by assuming a simple two-layer model. This suggests the existence of an immobile layer near the polymer-core interface, and such immobile layer may be originated from a higher crosslink density near the interface (heterogeneous crosslink effect) as well as from the chain adsorption at the core/shell interface. The $T_{\mathrm{g}}$ of hollow shells, which is a counterpart of free-standing films, also exhibits a similar $d$-dependence to that observed for the filled shells. This suggests that the heterogeneous crosslink effect 
dominates over the chain adsorption effect. Furthermore, the effect of unstable configuration, which is expected to become prominent with decreasing $d$, may also be responsible for the observed $T_{\mathrm{g}}$ behaviors. It is likely that such configurational effects on the glass transition behaviors are generally significant for nanosized polymer systems such as spin-coated thin films, ${ }^{50,51}$ though contradictory results have also been reported. ${ }^{52}$

The characteristic length scale of the glass transition has been evaluated by TMDSC for the filled shells. From the $d$-dependence of the ratio $\xi_{\mathrm{f}}\left(T_{\mathrm{g}}\right) / \xi_{\mathrm{b}}\left(T_{\mathrm{g}}\right)$, it has been concluded that the characteristic length decreases with decreasing $d$. This may concern the configurational nature of the crosslinked polymeric shell, but intuitive understanding in terms of the configurational entropy has not yet been achieved. Additionally, a weak dependence of $\xi\left(T_{\mathrm{g}}\right)$ on the average distance between crosslink units has been revealed. This indicates that the characteristic length scale is not directly determined by the inter-crosslink distance.

The surface of the shell for the present capsules is ragged; this suggests occurrence of microemulsion polymerization to some extent. We have already found that capsules with other crosslinked polymer shells such as poly(methyl methacrylate) show smooth surface morphology. Glass transition behaviors of such systems are now under investigation.

ACKNOWLEDGEMENT This work was supported by a Grant-in-Aid for Scientific Research (No. 18550107) from the Ministry of Education, Culture, Sports, Science and Technology of Japan. The authors thank Maruo Calcium Co., Japan, for supplying the calcium carbonate material (BS-P).

\section{REFERENCES AND NOTES}

1. Thurmond II, K. B.; Kowalewski, T.; Wooley, K. L. J Am Chem Soc 1997, 119, 6656.

2. Hotz, J.; Meier, W. Langmuir 1998, 14, 1031.

3. Radtchenko, I. L.; Sukhorukov, G. B.; Leporatti, S.; Khomutov, G. B.; Donath, E.;

Möhwald, H. J Colloid Interface Sci 2000, 230, 272.

4. Crespy, D.; Stark, M.; Hoffmann-Richter, C.; Ziener, U.; Landfester, K.

Macromolecules 2007, 40, 3122.

5. Sasaki, T.; Shimizu, M.; Wu, Y.; Sakurai, K. J Nanomater 2008, 2008, 185632.

6. Sasaki, T.; Kawagoe, S.; Mitsuya, H.; Irie, S.; Sakurai, K. J Polym Sci Part B: Polym

Phys 2006, 44, 2475.

7. Forrest, J. A.; Dalnoki-Veress, K. Adv. Colloid Interface Sci 2001, 94, 167. 
8. Forrest, J. A. Eur Phys J E 2002, 8, 261.

9. Keddie, J. L.; Jones, R. A. L.; Cory, R. A. Faraday Discuss 1994, 98, 219.

10. Keddie, J. L.; Jones, R. A. L.; Cory, R. A. Europhys Lett 1994, 27, 59.

11. Fryer, D. S.; Nealey, P. F.; de Pablo, J. J. Macromolecules 2000, 33, 6439.

12. Mattsson, J.; Forrest, J. A.; Börjesson, L. Phys Rev E 2000, 62, 5187.

13. Kawana, S.; Jones, R. A. L. Phys Rev E 2001, 63, 021501.

14. Pham, J. Q.; Green, P. F.; Macromolecules 2003, 36, 1665.

15. Roth, C. B.; McNerny, K. L.; Jager, W. F.; Torkelson, J. M. Macromolecules 2007, 40, 2568.

16. Arndt, M.; Stannarius, R.; Groothues, H.; Hempel, E.; Kremer, F. Phys Rev Lett 1997, 79, 2077.

17. Park, J.-Y.; McKenna, G. B. Phys Rev B 2000, 61, 6667.

18. Gauer, U.; Wunderlich, B. Macromolecules 1980, 13, 1618.

19. Sasaki, T.; Shimizu, A.; Mourey, T. H.; Thurau, C. T.; Ediger, M. D. J Chem Phys 2003, 119, 8730.

20. DeMaggio, G. B.; Frieze, W. E.; Gidley, D. W.; Zhu, M.; Hristov, H. A.; Yee, A. F. Phys Rev Lett 1997, 78, 1524.

21. Fukao, K.; Miyamoto, Y. Phys Rev E 2000, 61, 1743.

22. Kim, J. H.; Jang, J.; Zin, W.-C. Langmuir 2001, 17, 2703.

23. Fryer, D. S.; Peters, R. D.; Kim, E. J.; Tomaszewski, J. E.; de Pablo, J. J.; Nealey, P. F.; White, C. C.; Wu, W. Macromolecules 2001, 34, 5627.

24. Ellison, C. J.; Torkelson, J. M. Nat Mater 2003, 2, 695.

25. Ngai, K. L.; Rizos, A. K.; Plazek, D. J. J Non-Cryst Solids 1998, 235-237, 435.

26. de Gennes, P. G. Eur Phys J E 2000, 2, 201.

27. Long, D.; Lequeux, F. Eur Phys J E 2001, 4, 371.

28. Cicerone, M. T.; Blackburn, F. R.; Ediger, M. D. Macromolecules 1995, 28, 8224.

29. Böhmer, R.; Hinze, G.; Diezemann, G.; Geil, B.; Sullescu, H. Europhys Lett 1996, 36, 55.

30. Tracht, U.; Wilhelm, M.; Heuer, A.; Feng, H.; Schmidt-Rohr, K.; Spiess, H. W. Phys Rev Lett 1998, 81, 2727.

31. Schawe, J. E. K. Thermochim Acta 1995, 261, 183.

32. Hemmpel, E.; Hensel, A.; Schick, C.; Donth, E. J Phys Chem B 2000, 104, 2460.

33. Adam, G.; Gibbs, J. J Chem Phys 1965, 43, 139.

34. Sasaki, T.; Kitagawa, T.; Sato, S.; Irie, S.; Sakurai, K. Polym J 2005, 37, 434.

35. Sanetra, R.; Kolarz, B. N.; Wlochowicz, A. Polymer 1985, 26, 1181.

36. Weyer, S.; Merzlyakov, M.; Schick, C. Thermochim Acta 2001, 377, 85. 
37. Schick, C.; Merzlyakov, M.; Hensel, A. J Chem Phys 1999, 111, 2695.

38. Crider, P. S.; Majewski, M. R.; Zhang, J.; Oukris, H.; Israeloff, N. E. J Chem Phys 2008, 128, 044908.

39. Bogoslovov, R. B.; Roland, C. M.; Ellis, A. R.; Randall, A. M.; Robertson, C. G. Macromolecules 2008, 41, 1289.

40. Sharp, J. S.; Forrest, J. A. Phys Rev Lett 2003, 91, 235701.

41. Sharp, J. S.; Forrest, J. A. Phys Rev E 2003, 67, 031805.

42. Akabori, K.; Tanaka, K.; Nagamura, T.; Takahara, A.; Kajiyama, T. Macromolecules 2005, 38, 9735.

43. Grohens, Y.; Sacristan, J;. Hamon, L.; Reinecke, H.; Mijangos, C.; Guenet, J. M. Polymer 2001, 42, 6419.

44. Wang, X.; Zhou, W. Macromolecules 2002, 35, 6747.

45. Sasaki, T.; Uchida, T.; Sakurai, K. J Polym Sci Part B: Polym Phys 2006, 44, 1958.

46. Kannurpatti, A. R.; Anderson, K. J.; Anseth, J. W.; Bowman, C. N. J Polym Sci Part B: Polym Phys 1997, 35, 2297.

47. Scott, T. F.; Cook, W. D.; Forsythe, J. S. Eur Polym J 2002, 38, 705.

48. Alves, N. M.; Gómez Ribelles, J. L.; Mano, J. F. Polymer 2005, 46, 491.

49. The previous data for crosslinked polystyrenes in ref 45 show higher characteristic length than $L$; this is partially due to an inappropriate estimation of DVB content, in which impurities contained in DVB were not taken into account.

50. Brûlet, A.; Boué, F.; Menelle, A.; Cotton, J. P. Macromolecules 2000, 33, 997.

51. Tsui, O. K. C.; Wang, Y. J.; Lee, F. K.; Lam, C.-H.; Yang, Z. Macromolecules 2008, 41, 1465.

52. Fakhraai, Z.; Sharp, J. S.; Forrest, J. A. J Polym Sci Part B: Polym Phys 2004, 42, 4503. 
Table 1. Thickness of the prepared capsules

\begin{tabular}{lccc}
\hline Sample & $X_{\mathrm{c}}(\%)$ & $d_{\mathrm{f}}(\mathrm{nm})^{\mathrm{a}}$ & $d_{\mathrm{h}}(\mathrm{nm})^{\mathrm{b}}$ \\
\hline A & 0.61 & $78 \pm 13$ & $115 \pm 43$ \\
B & 1.48 & $25 \pm 14$ & $60 \pm 20$ \\
C & 1.55 & $68 \pm 23$ & $84 \pm 21$ \\
D & 2.49 & $49 \pm 18$ & $68 \pm 24$ \\
E & 3.54 & $45 \pm 17$ & $64 \pm 26$ \\
F & 4.60 & $118 \pm 21$ & $129 \pm 28$ \\
G & 4.61 & $39 \pm 10$ & $59 \pm 12$ \\
H & 4.62 & $76 \pm 16$ & $84 \pm 20$ \\
I & 4.63 & $20 \pm 6$ & $37 \pm 6$ \\
\hline
\end{tabular}

${ }^{a}$ Shell thickness of the filled core/shell capsule.

b Shell thickness of the hollow capsule.

Table 2. Characteristic length and glass transition temperature of the interface layer of the filled shells

\begin{tabular}{llll}
\hline$X_{\mathrm{c}}(\%)$ & $\xi_{\mathrm{f}}\left(T_{\mathrm{g}}\right)(\mathrm{nm})$ & $T_{\mathrm{g}}^{\mathrm{i}}(\mathrm{K})$ & $T_{\mathrm{g}}^{\mathrm{i}} / T_{\mathrm{g}}^{\mathrm{f}}$ \\
\hline 0.61 & $2.33 \pm 0.20$ & 449.9 & 1.19 \\
1.55 & $1.83 \pm 0.12$ & 475.3 & 1.24 \\
2.49 & $1.54 \pm 0.08$ & 498.2 & 1.29 \\
3.54 & $1.22 \pm 0.07$ & 531.3 & 1.37 \\
4.61 & $1.02 \pm 0.05$ & 561.0 & 1.44 \\
\hline
\end{tabular}




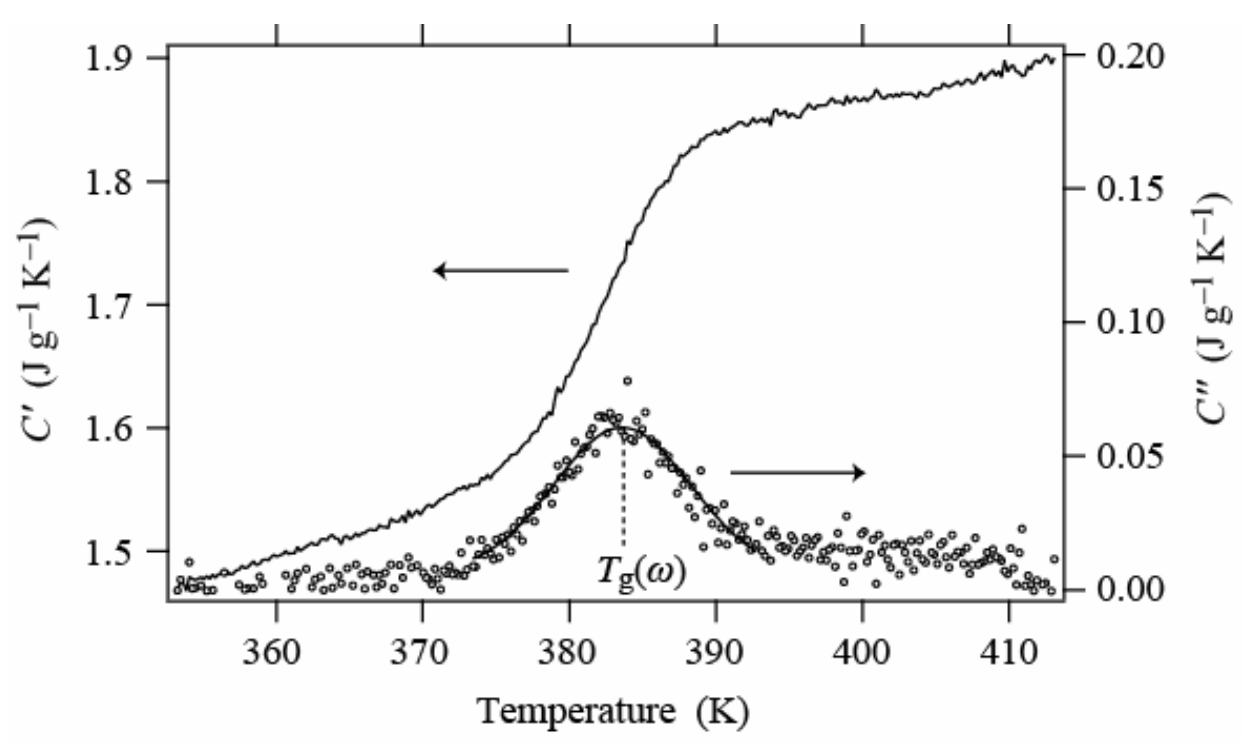

Figure 1. Typical profiles for the storage and loss heat capacities of crosslinked polystyrene (bulk sample with $X_{\mathrm{c}}=1.55 \%$ ). The fitted curve for $C^{\prime \prime}(T)$ according to eq 5 is also shown.

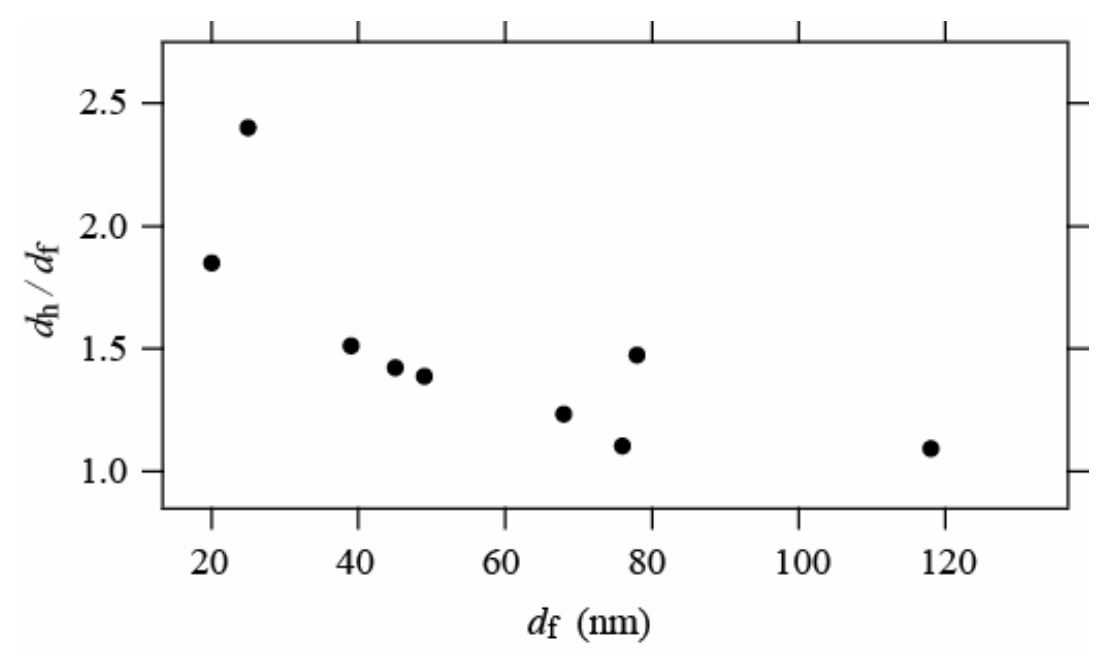

Figure 2. The ratio $d_{\mathrm{h}} / d_{\mathrm{f}}$ plotted against $d_{\mathrm{f}}$. 
(a)

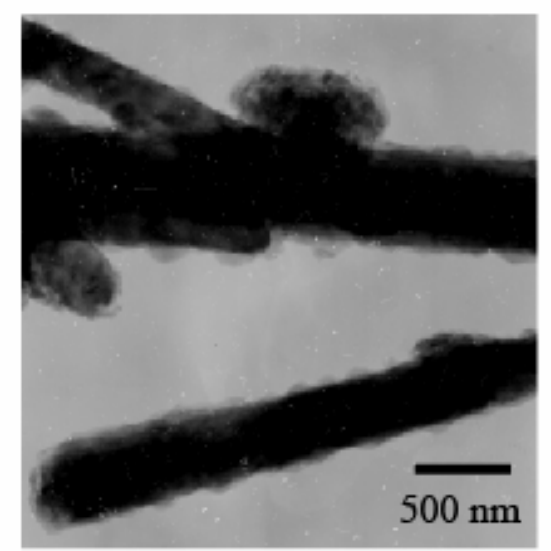

(c)

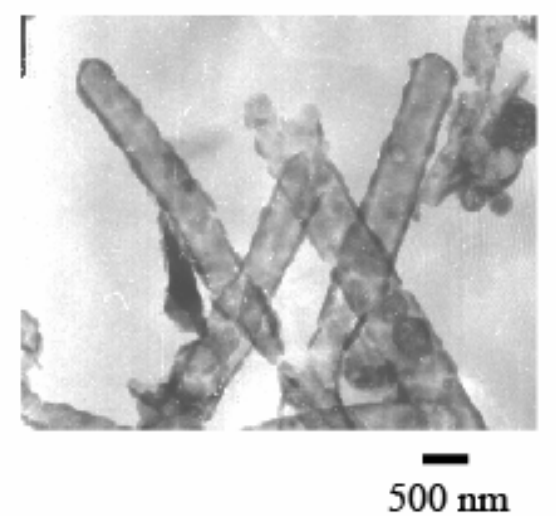

(b)

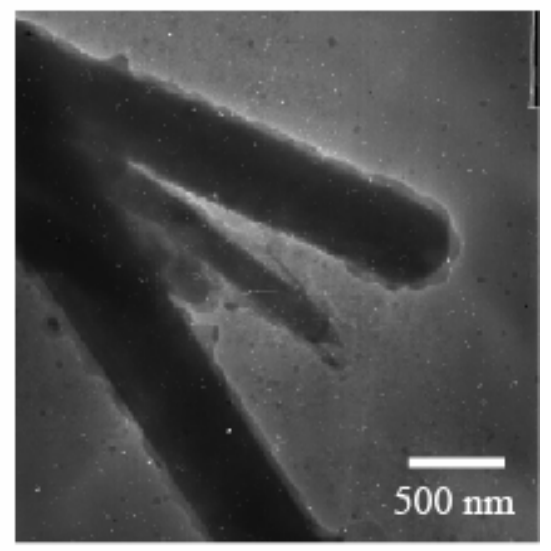

Figure 3. Typical transmission electron micrographs for rodlike core/shell capsules of (a): $X_{\mathrm{c}}=1.48 \%$, (b): $X_{\mathrm{c}}=3.54 \%$, and (c): hollow capsules of $X_{\mathrm{c}}=0.61 \%$. 


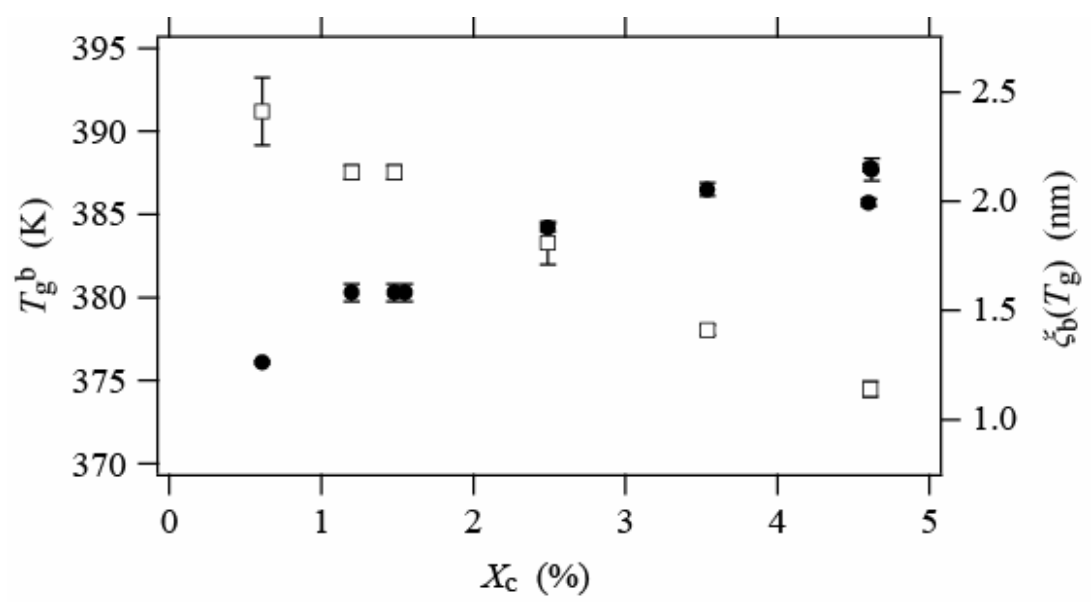

Figure 4. Glass transition temperature $T_{\mathrm{g}}$ (filled circles) and characteristic length $\xi\left(T_{\mathrm{g}}\right)$ (open squares) for the reference bulk samples plotted against $X_{\mathrm{c}}$.

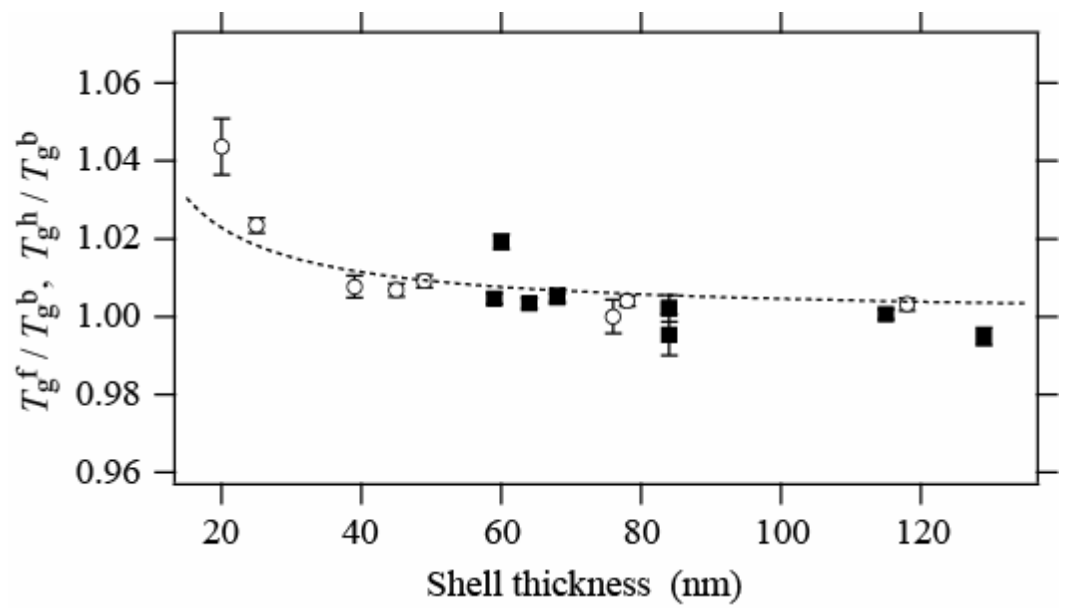

Figure 5. The ratio of the glass transition temperature of the shells to that of the bulk plotted against the shell thickness. The open circles indicate $T_{\mathrm{g}}{ }^{\mathrm{f}} / T_{\mathrm{g}}{ }^{\mathrm{b}}$, and the filled squares indicate $T_{\mathrm{g}}^{\mathrm{h}} / T_{\mathrm{g}}^{\mathrm{b}}$. The dotted curve indicates the fitting result according to eq 6. 


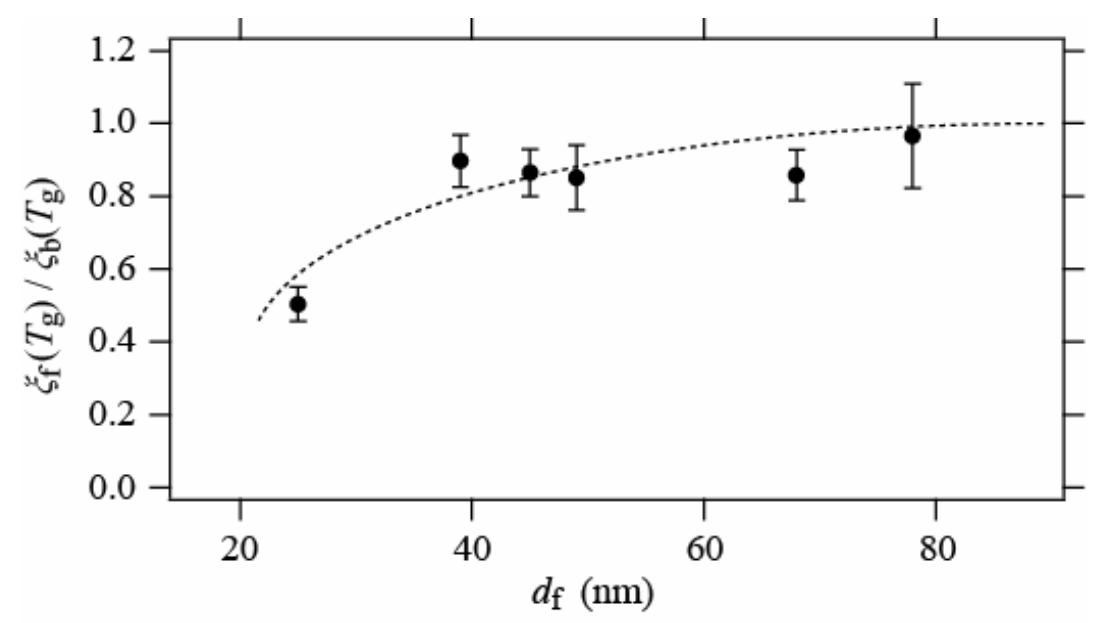

Figure 6. The ratio of the characteristic length for the filled shell to that of the bulk, $\xi_{\mathrm{f}}\left(T_{\mathrm{g}}\right) / \xi_{\mathrm{b}}\left(T_{\mathrm{g}}\right)$ plotted against $d_{\mathrm{f}}$. The dotted curve is a guide for the eye.

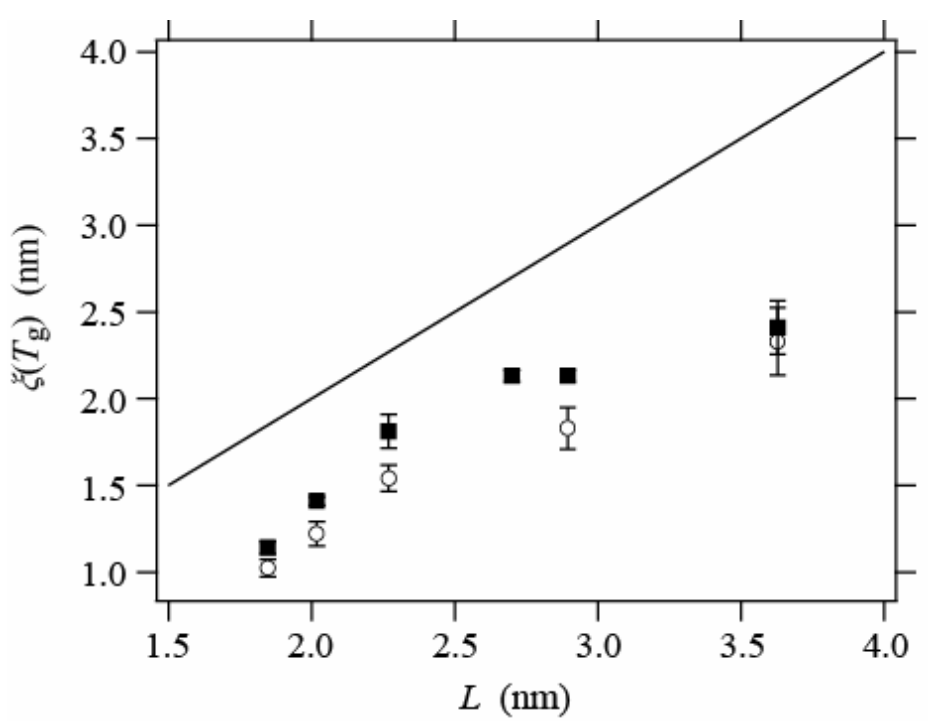

Figure 7. Characteristic length $\xi\left(T_{\mathrm{g}}\right)$ plotted against the average distance between the nearest neighboring crosslink units $L$. The open circles indicate $\xi_{\mathrm{f}}\left(T_{\mathrm{g}}\right)$, and the filled squares indicate $\xi_{\mathrm{b}}\left(T_{\mathrm{g}}\right)$. The solid line indicates $\xi\left(T_{\mathrm{g}}\right)=L$. 